\title{
Application research of micro and nano bubbles in water pollution control
}

\author{
Chang Liu ${ }^{12}$, Yuchao Tang ${ }^{12}$ \\ ${ }^{1}$ School of Environment and Energy Engineering, Anhui JianZhu University, 230601Hefei, China \\ ${ }^{2}$ Key Laboratory of Huizhou Architecture in Anhui Province, Anhui JianZhu University, 230601Hefei, China
}

\begin{abstract}
The article describes the characteristics of micro-nano bubbles in the water for a long time, large specific surface area, high gas-liquid mass transfer efficiency, high interface potential and free radical generation. The generation of micro-nano bubbles is introduced, such as dissolved gas release method and dispersion. Air method, electrolysis method, etc. It mainly summarizes the research and application of micro-nano bubbles in surface water treatment, groundwater remediation, industrial wastewater treatment and enhanced ozone treatment, as well as practical applications in ship operation, metal surface degreasing and fruit and vegetable growth.
\end{abstract}

\section{Introduction}

Generally speaking, bubbles with a particle size between 10 and $50 \mu \mathrm{m}$ are micro-bubbles, bubbles with a particle size of less than $200 \mathrm{~nm}$ are called nano-bubbles ${ }^{[1]}$, and bubbles with a particle size between the two are called micro-nano bubbles. The initial micro-nano bubbles are mainly for the sterilization of aquatic products such as oysters. With the continuous development of technology, micro-nano bubbles have gradually been applied in other fields, and more and more people are paying attention.

\section{Characteristics of micro-nano bubbles}

\subsection{Long stay time}

Due to the small diameter of the micro-nano bubbles, the buoyancy in the water is less than the buoyancy of the larger diameter bubbles.

\subsection{Large specific surface area}

It can be seen from the $\mathrm{S} / \mathrm{V}=3 / \mathrm{r}$ formula that the bubble volume is constant, and the specific surface area of the bubble is inversely proportional to its radius, that is, the small radius of the bubble makes the specific surface area larger. Therefore, the specific surface area of the micro-nano bubbles is large.

\subsection{High gas-liquid mass transfer efficiency}

The mass transfer efficiency of a gas is determined by the specific surface area of the gas and liquid, and the specific surface area of the gas and liquid is determined by the volume and diameter of the gas in the liquid ${ }^{[2]}$. The smaller the gas diameter, the greater the surface tension it receives. Under the influence of surface tension, the micro-nano bubbles begin to shrink and the pressure inside the bubbles rises. When the bubble is continuously reduced to a certain extent, the pressure inside the bubble will approach an infinite value, so that the micro-nano bubble dissolves in water or ruptures at the surface of the water ${ }^{[3]}$.

\subsection{Producing free radicals}

The micro-nano bubble has a gas-liquid interface in the liquid. When the bubble collapses, the disappearance of the gas-liquid interface causes the energy of the high concentration of positive and negative ions accumulated at the interface to be released, thereby generating a large amount of hydroxyl radicals. The hydroxyl radical is highly oxidizing, and it can degrade pollutants such as phenol which are difficult to be decomposed under normal conditions, thereby purifying the water.

\subsection{High interface potential}

The potential difference produced by the electric double layer on the surface of the bubble is usually expressed by the zeta potential, which represents the interfacial potential of the micro-nano bubble. Ushikubo ${ }^{[4]}$ et al. have shown that the zeta potential of air micro-nano bubbles is between $-20 \mathrm{mV}$ and $-17 \mathrm{mV}$, while the zeta potential of oxygen micro-nano bubbles is between $-45 \mathrm{mV}$ and -34 $\mathrm{mV}$.

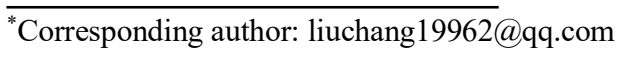




\section{Micro-nano bubble generation}

\subsection{Dissolved gas release method}

The dissolved gas release method is mainly achieved by the increase and decrease of pressure. First, a certain pressure is applied to the gas to dissolve it in the liquid, and then the pressure is lowered, and the gas previously dissolved in the liquid is released in the form of micro-nano. The method is simple in operation and low in energy consumption, and the foam generating device based on this technology has entered the application stage.

\subsection{Dispersed air method}

This method mainly involves repeatedly shearing and breaking the bubbles by stirring and high-speed shearing to form micro-nano bubbles in the water. According to different shearing materials, the method can be further divided into the following three types: microplate method, mechanical method and jet method.

The microplate method compresses air through a specific plate with a certain size of micropores. When the gas passes through the micropores, it is sheared by the micropores to form micro-nano bubbles. The mechanical method cuts the gas to the micro-nano level by rapid rotation of the impeller. The jet method is divided into two types: air compressor injection type and self-priming type. The former mainly uses a compressor to inject air into the suction chamber, and the gas and liquid are mixed and then jetted at high speed to generate micro-nano bubbles in the state of gas-liquid turbulence. The self-priming type mainly uses a high-speed jet to form a negative pressure state in the suction chamber, thereby allowing air to enter the suction chamber.

\subsection{Electrolysis}

The electrolysis method mainly generates microbubbles on both poles of the electrode by energizing the water. However, this method has disadvantages such as large energy consumption, consumption of electrodes during electrolysis, and a small amount of bubbles generated by electrolysis. In addition to the above three methods, conventional methods include ultrasonic method, high temperature method, chemical method, and the like.

\section{Application of micro-nano bubbles in water treatment direction}

\subsection{Micro-nano bubble treatment of surface water}

Micro-nano bubbles can greatly increase the dissolved oxygen content in water, and enhance the oxidation effect on surface water. The Incheon International Airport in South Korea is an example. It has achieved good results by using micro-nano bubbles to control its surface water pollution.

Yang Qiang et al. performed micro-nano bubble treatment on a river channel in Hangzhou. It was found that the dissolved oxygen in the water treated by micro-nano bubbles increased significantly, and the concentrations of ammonia nitrogen, total nitrogen, TDS and total phosphorus decreased significantly. Permanganic acid The potassium index also decreased by $6.78 \mathrm{mg} / \mathrm{L}$. Zhang Kuixing et al. used a bubble generating device with a bubble diameter of $200-4000 \mu \mathrm{m}$ to treat a heavily polluted swell in the Pearl River. The dissolved oxygen content in the water body increased significantly. The four monitoring results showed that the BOD removal rate is between $70 \%$ and $80 \%$, the removal rates of ammonia nitrogen, SS and animal oil are all above $80 \%$, and the total phosphorus removal rate is basically $80 \%$;

\subsection{Micro-nano bubble treatment of groundwater}

The problem of groundwater pollution in China is becoming more and more serious. Nitrate, petroleum products and bacterial viruses all cause different degrees of pollution to groundwater. Micro-nano bubbles are used to treat groundwater, so that dissolved oxygen content is significantly increased and pollutants are removed.

Li Hengzhen ${ }^{[5]}$ et al. experiments have proved that micro-nano bubbles can increase the dissolved oxygen content in groundwater and reduce the content of pollutants in groundwater. $\mathrm{Li}{ }^{[6]}$ et al. proved that micro-nano bubbles can significantly increase the oxygen mass transfer rate and accelerate the removal of groundwater pollutants. The dissolved oxygen transport rate of oxygen microbubbles is the fastest, nearly 125 times faster than air microbubbles, and the highest dissolved oxygen peak. It is nearly three times larger than air micro-nano bubbles, and the dissolved oxygen enhances durability for the longest, 16 times longer than air micro-nano bubbles. $\mathrm{Li}^{[7]}$ et al. found that when the particle size of the bubble is between $500 \mathrm{~nm}$ and $100 \mu \mathrm{m}$, the dissolved oxygen increases faster and the dissolved oxygen peak is higher. Adding surfactant in water can reduce the bubble size, reduce the oxygen transfer efficiency, and prolong the bubble stagnation time, increase bubble interface charge. $\mathrm{Hu}{ }^{[8]}$ et al. found that micro-nano bubbles can significantly enhance the efficiency of ozone transfer. In laboratory conditions, the impact on groundwater organic pollutants is significant, which can greatly improve the treatment efficiency. Field experiments have been carried out on a lead-contaminated site in Japan using ozone microbubbles, with a total removal rate of $99 \%$.

\subsection{Micro-nano bubble treatment wastewater}

The microbubble has a high zeta potential and a large specific surface area, so that the contact time between the bubble and the suspended matter becomes longer, and the adhesion efficiency is improved. Therefore, the combination of microbubble technology and coagulation technology can enhance the removal of pollutants in wastewater ${ }^{[9]}$.

Deng ${ }^{[10]}$ et al. used a combination of microbubble air flotation technology and T-tube oil-water separation 
technology to treat oily wastewater. The final mass of the oil was reduced from $38-350 \mathrm{mg} / \mathrm{L}$ to $12-61 \mathrm{mg} / \mathrm{L}$. The device also reduces production costs. Liu ${ }^{[11]}$ et al. found in the pretreatment of printing and dyeing wastewater that the removal rate of COD, color and oil by coagulation microbubble flotation is higher than that of coagulation conventional bubble flotation, and the amount of coagulant required is less. The pretreatment time is shorter.

\subsection{Micro-nano bubbles strengthen ozone}

Ozone is highly oxidizing and is often used in wastewater treatment ${ }^{[12]}$. However, the treatment of some organic substances by ozone alone is not ideal. Therefore, the application of ozone instead of air to micro-nano bubble technology can not only improve the mass transfer rate of ozone in water. Make it more fully utilized, and also strengthen its oxidizing ability and accelerate the removal of pollutants in water.

Chu ${ }^{[13]}$ et al. found that micro-nano bubble ozonation can reduce the sludge. Compared with the ordinary bubble ozonation process, the former greatly increased the utilization rate of ozone, up to $99 \%$, while the latter was around $80 \mathrm{~min}$. The utilization rate of ozone has dropped from $94 \%$ to $72 \%$. Chu ${ }^{[14]}$ et al. also used micro-nano bubble ozone oxidation process to treat textile wastewater. The experiment found that the micro-nano bubble ozone oxidation process has a COD removal rate of $70 \%$, while the ordinary bubble ozone oxidation technology has a COD removal rate of only $50 \%$. Comparing the decolorization effect, the time required to remove $80 \%$ of the chromaticity of the microbubble process and the ordinary bubble process were $140 \mathrm{~min}$ and $280 \mathrm{~min}$, respectively. In addition, experiments have also shown that microbubbles can also improve the oxidation properties of textile wastewater. Liu ${ }^{[15]}$ et al. used ozone, air and oxygen microbubble flotation process to treat carbon waste water. The experiment proved that ozone microbubbles can produce more hydroxyl radicals, can remove more organic matter, and combine ozone microbubbles with coagulation sedimentation technology. The removal rates of stupid and pyridine were higher than the latter two, and the removal rates of pyridine were 4.5 times and 1.7 times higher, respectively, and benzene was 3.6 times and 1.5 times higher, respectively.

\section{Reference}

1. Agarwal A, Ng W J, Liu Y. Principle and applications of microbubble and nanobubble technology for water treatment[J]. Chemosphere, 2011, 84(9):1175-1180.

2. Burris V, Little J. Bubble dynamics and oxygen transfer in a hypolimnetic aerator[J]. Water Science \& Technology, 1998, 37(2):293-300.
3. Takahashi M, Kawamura T, Yamamoto Y, et al. Effect of Shrinking Microbubble on Gas Hydrate Formation[J]. The Journal of Physical Chemistry B, 2003, 107(10):2171-2173.

4. Ushikubo F Y, Furukawa T, Nakagawa R, et al. Evidence of the existence and the stability of nano-bubbles in water[J]. Colloids and Surfaces A: Physicochemical and Engineering Aspects, 2010, 361(1-3):31-37.

5. Heng-Zhen L I, Li-Ming $\mathrm{H}$ U, Hong-Bo $\mathrm{X}$. Application of micro-nano bubble technology in remediation of polluted groundwater[J]. Chinese Journal of Geotechnical Engineering, 2015, 37:115-120.

6. Li H, Hu L, Song D, et al. Subsurface Transport Behavior of Micro-Nano Bubbles and Potential Applications for Groundwater Remediation[J]. International Journal of Environmental Research and Public Health, 2013, 11(1):473-486.

7. $\mathrm{Li} \mathrm{H}, \mathrm{Hu} \mathrm{L}$, Song $\mathrm{D}$, et al. Characteristics of Micro-Nano Bubbles and Potential Application in Groundwater Bioremediation[J]. Water Environment Research, 2014, 86(9):844-851.

8. $\mathrm{Hu} \mathrm{L}$, Xia $\mathrm{Z}$. Application of Ozone Micro-Nano-Bubbles to Groundwater Remediation[J]. Journal of Hazardous Materials, 2017, 342.

9. Guet S, Ooms G. FLUID MECHANICAL ASPECTS OF THE GAS-LIFT TECHNIQUE[J]. Annual Review of Fluid Mechanics, 2006, 38(1):225-249.

10. Xiaohui D, Jingyu X, Yingxiang W, et al. Research on the technology of oil removal by dynamic state micro-bubbles flotation[J]. Industrial Water Treatment, 2011, 31(4):89-90.

11. Liu S. Effect of Micro-Bubbles on Coagulation Floation Process of Dyeing Wastewater[J]. Separation Purification Tech. 2010, 71.

12. Perkowski J, Kos L, Ledakowicz S. Application of Ozone in Textile Wastewater Treatment[J]. Ozone Science and Engineering, 1996, 18(1):73-85.

13. Chu L B, Yan S T, Xing X H, et al. Enhanced sludge solubilization by microbubble ozonation[J]. Chemosphere, 2008, 72(2):0-212.

14. Chu L B, Xing X H, Yu A F, et al. Enhanced treatment of practical textile wastewater by microbubble ozonation[J]. Process Safety \& Environmental Protection, 2008, 86(5):389-393.

15. Liu S, Wang Q, Sun T, et al. The effect of different types of micro-bubbles on the performance of the coagulation flotation process for coke waste-water[J]. Journal of Chemical Technology \& Biotechnology, 2012, 87(2):206-215. 